\title{
Image Quality Assessment
}

\author{
Susan Farnand \\ Rochester Institute of Technology \\ 54 Lomb Memorial Drive \\ Rochester, New York 14623-5603 \\ farnand@cis.rit.edu
}

Frans Gaykema

Océ Technologies BV

St. Urbanusweg 43

5914 CA Venlo, The Netherlands

frans.gaykema@oce.com

Images represent a powerful means to convey information. This transfer may occur from one human to another through a system or device, or directly from a system or device to human observers, either for the purpose of a specific task or for enjoyment. Whatever the purpose, the quality of these images is an issue that is of profound interest to almost any developer working on imaging technologies. Image quality is of obvious interest when an image is rendered by a device on some medium for viewing. But, image quality is impacted not only by that device and medium, but also by the other components in the imaging chain. For image capture, for example, transmission and processing of the images at different stages is important to consider as well. Developers must consider how the perceived image quality relates to the technical system parameters and technology characteristics. This proves to be a complex and challenging task because of the indispensable contribution of the human observer. The focus of this special section is on both objective and subjective methods to evaluate and assess image quality as experienced by human observers.

Imaging has a long history, punctuated by major development milestones through the centuries. The invention of oil paint allowed an increase in image-tone scale; the invention of optical lenses allowed for magnification of images useful to biological and astronomical research; the invention of the photographic process allowed images to be stored and shared. Now we are in an era of digital imaging in which we are experiencing explosive growth in the capabilities and applications of imaging technology. Developments in computer-processing power, data-transmission speed, compression techniques, wireless networks, and mobile imaging devices expand the ways we create and share imagery. In just a few short years, we have experienced the introduction and increasing use of cell-phone cameras, print-on-demand photobooks, high-dynamic-range displays, electronic paper, and 3-D capabilities in printing, display, and cinema. Digital imaging has become woven into the fabric of our society.

Research on image-quality evaluation methods has evolved alongside the advancement of digital imaging. New imaging technologies and applications require an assessment of the level of image quality achieved. It may be of paramount importance to engineers and scientists developing new systems to know whether these systems yield im- ages of an acceptable quality for their target market or havea sufficient competitive edge in quality to justify a price premium. It is consequently essential to have metrics that quantify the effects of system changes on the perceived image quality of the output. Although objective evaluation techniques themselves have benefited greatly from the advancements in digital-imaging technologies, there is one aspect that has remained in the subjective realm of image-quality evaluation: the human factor. It is critical that the objective evaluation methods correlate well with the users subjective perception of quality, thus necessitating methods to establish or validate such relationships.

The papers in this special section provide an extensive, though perhaps not exhaustive, overview of different approaches to characterize the perceptual quality of images from a human customer perspective. They may be considered examples of the current state of image-quality research, ranging from descriptions of possible techniques to evaluation of images produced using new technologies. This special section includes, for example, a paper describing a soft-copy ruler technique for the general evaluation of images. It includes papers evaluating on-demand photobooks and the effects of digital in-painting, as well as several papers addressing image-quality issues for applications using high-dynamic-range imaging. This special section also offers papers describing metrics for specific image characteristics or defects, as well as papers covering general metrics for technologies as old as printing, and as new as laser range scanning. There are papers proposing image-enhancement techniques for printing and for ultrasound. Finally, there are several papers that address aspects of image-content effects on the perception and measurement of image quality.

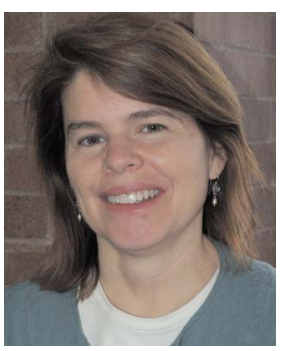

Susan Farnand is a research scientist at the Rochester Institute of Technology. She graduated from Cornell University with a BS in engineering and from the Rochester Institute of Technologys with a masters degree in imaging science. She worked for many years at Eastman Kodak Company on assignments involving electrophotography and imaging science, before leaving to join RIT as a research scientist. With a focus on human color vision and psycho- 
physics, she is involved with investigations into fine art reproduction, visual attention, gloss appearance, printer performance evaluation, and various aspects of image-quality assessment. She also participates in International Standards efforts and the IS\&T professional society, including serving as cochair of the Image Quality and System Performance Conference of the IS\&T/SPIE Electronic Imaging symposium since 2007.

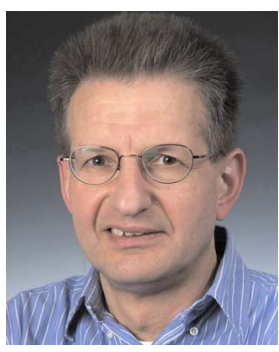

Frans Gaykema received his MSc degree in physics from the Eindhoven University of Technology in 1985. He joined the R\&D laboratories of Océ-Technologies in Venlo, where he is involved in color and imagequality characterization and evaluation research. $\mathrm{He}$ is a member of the Society for Information Display and IS\&T, notably involved with the organization of the Image Quality and System Performance Conference as part of the IS\&T/SPIE Electronic Imaging symposium, where he serves as a conference cochair since 2007. Frans has also participated in International Standards efforts for many years, with a focus in the area of printed image quality. 